\title{
ON GRADED BIALGEBRA DEFORMATIONS
}

\author{
YU DU, XIAO-WU CHEN AND YU YE* \\ Department of Mathematics \\ University of Science and Technology of China \\ Hefei 230026, Anhui, P. R. China \\ USTC Shanghai Institute for Advanced Studies \\ Shanghai, 201315, P.R.China
}

\begin{abstract}
We introduce the graded bialgebra deformations, which explains Andruskiewitsch-Schneider's liftings method. We also relate this graded bialgebra deformation with the corresponding graded bialgebra cohomology groups, which is the graded version of the one due to Gerstenhaber-Schack.
\end{abstract}

\section{INTRODUCTION}

The classification of finite-dimensional pointed Hopf algebras is a basic problem in the theory of Hopf algebras. It is well-known that any pointed Hopf algebra $H$ has a coradical filtration, with respect to which one associates a coradically-graded Hopf algebra gr $H$. Following Andruskiewitsch and Schneider, the classification problem can be divided into two parts. One is the classification of all coradically-graded pointed Hopf algebras. The other is to find all possible pointed Hopf algebras $H$ with gr $H$ isomorphic to a given coradically-graded pointed Hopf algebra. The second part is just the lifting method in [1] and 2]. One of our motivations is to relate the lifting method with certain bialgebra deformation theory.

The deformation theory for algebras is initiated by Gerstenhaber in [4, and its analogue for bialgebras appeared first in [5] (also see [6] and [9]). Inspired by the graded algebra deformation theory in [3], we develop in this paper the theory of graded bialgebra deformations and their corresponding cohomology groups. Moreover this deformation theory can be used to explain Andruskiewitsch-Schneider's lifting method.

Key words and phrases. Graded Bialgebras, Liftings, Deformations.

* The corresponding author.

Supported in part by the National Natural Science Foundation of China (Grant No. 10271113) and the Europe Commission AsiaLink project "Algebras and Representations in China and Europe" ASI/B7-301/98/679-11.

E-mail: ydu@mail.ustc.edu.cn, xwchen@mail.ustc.edu.cn, yeyu@ustc.edu.cn. 
The paper is organized as follows. In section 2, first we recall the notion of liftings and introduce the graded bialgebra deformations, and we show that the lifting is just the same as the graded bialgebra deformation in the sense of Theorem 2.2. The graded-rigid bialgebras are also studied, see Corollary 2.3 and Corollary 2.4. In section 3, we introduce the notion of graded "hat" bialgebra cohomology groups for graded bialgebras, which controls the graded bialgebra deformations, see Theorem 3.3.

\section{Liftings AND GRADED BIALGEBRA DEFORMATIONS}

We will work on a base field $\mathbb{K}$. All unadorned tensors are over $\mathbb{K}$. We refer the notion of graded bialgebras and filtered bialgebras to [11], the notion of graded linear maps to [8].

2.1. Let us recall Andruskiewitsch-Schneider's liftings method, for more details, see 2. Note that the liftings defined here is a slight generalization.

Throughout, $B=\oplus_{n \geq 0} B_{(n)}$ will be a graded bialgebra over $\mathbb{K}$, with identity element $1_{B}$, multiplication map $m$, counit $\varepsilon$, and comultiplication $\Delta$. Then $B$ has a natural Hopf filtration

$$
B_{0} \subseteq B_{1} \subseteq \cdots \subseteq B_{n} \subseteq \cdots
$$

where $B_{n}=\oplus_{j \leq n} B_{(j)}$ for any $n \geq 0$.

A lifting of the graded bialgebra $B$ is a filtered bialgebra structure, denoted by $U$, on the underlying filtered vector space $B$ with the above filtration such that

$$
\operatorname{gr} U=B
$$

as graded bialgebras, where $\operatorname{gr} U$ is the graded bialgebra associated to the filtered bialgebra $U(11$, p.226). (By $\operatorname{gr} U=B$, we use the natural identification of the underlying space $\operatorname{gr} U$ with $B$, that is $B_{n} / B_{n-1} \cong B_{(n)}$ for each $n \geq 0$.)

For any lifting $U$ of the graded bialgebra $B$, it follows from the definition that $U$ and $B$ have the same identity element and the counit. Therefore, to give a lifting $U$, we just need to define the multiplication $m_{U}$ and comultiplication $\Delta_{U}$.

Two liftings $U, V$ of the graded bialgebra $B$ are said to be equivalent, if there is filtered bialgebra isomorphism $\theta: U \longrightarrow V$ such that $\operatorname{gr} \theta=\operatorname{Id}_{B}$, where $\operatorname{gr} \theta$ is the graded morphism associated to $\theta$, and here again we use the identifications $\operatorname{gr} U=B$ and $\operatorname{gr} V=B$ (as graded bialgebras).

Denote by

$$
\operatorname{Lift}(B)
$$

the set of equivalent classes of all the liftings of the graded bialgebra $B$. 
2.2. In this section, we will study graded bialgebra deformations of the graded bialgebra $B=\oplus_{n \geq 0} B_{(n)}$.

Let $l \in \mathbb{N} \cup\{+\infty\}$. Consider the space $B[t] /\left(t^{l+1}\right)$, which is viewed as a free module over $\mathbb{K}[t] /\left(t^{l+1}\right)$, and also a graded $\mathbb{K}$-space with $\operatorname{deg} t=1$ and $\operatorname{deg} b=n$, if $b \in B_{(n)}$. If $l=+\infty$, then $B[t] /\left(t^{l+1}\right)$ means $B[t]$ and $\mathbb{K}[t] /\left(t^{l+1}\right)$ means $\mathbb{K}[t]$.

An $l$-th level graded bialgebra deformation of $B$ consists of

$$
m_{t}^{l}:(B \otimes B)[t] /\left(t^{l+1}\right) \longrightarrow B[t] /\left(t^{l+1}\right)
$$

and

$$
\Delta_{t}^{l}: B[t] /\left(t^{l+1}\right) \longrightarrow(B \otimes B)[t] /\left(t^{l+1}\right)
$$

which are $\mathbb{K}[t] /\left(t^{l+1}\right)$-linear and homogeneous maps of degree zero such that

(i) $B[t] /\left(t^{l+1}\right)$ is a bialgebra over $\mathbb{K}[t] /\left(t^{l+1}\right)$ with identity element $1_{B}$, multiplication $m_{t}^{l}$, counit $\varepsilon_{t}^{l}$ and comultiplication $\Delta_{t}^{l}$, where the counit $\varepsilon_{t}^{l}: B[t] /\left(t^{l+1}\right) \longrightarrow \mathbb{K}[t] /\left(t^{l+1}\right)$ is given by $\varepsilon_{t}^{l}\left(b t^{j}\right)=\varepsilon(b) t^{j}$, $b \in B, 0 \leq j \leq l$

(ii) $m_{t}^{l} \equiv m \otimes \operatorname{Id}_{\mathbb{K}[t] /\left(t^{l+1}\right)}$ and $\Delta_{t}^{l} \equiv \Delta \otimes \operatorname{Id}_{\mathbb{K}[t] /\left(t^{l+1}\right)} \bmod (t)$, where $m$ and $\Delta$ are the multiplication and comultiplication of $B$, respectively.

Denote by $\left(B[t] /\left(t^{l+1}\right), m_{t}^{l}, \Delta_{t}^{l}\right)$ the above $l$-th level graded bialgebra deformation.

From now on, we will abbreviate $l$-th level graded bialgebra deformations as $l$-deformations, and $+\infty$-deformations will be referred simply as deformations. Denote by $\mathcal{E}^{l}(B)$ the set of all $l$-deformations of the graded bialgebra $B$, and $\mathcal{E}^{+\infty}(B)$ is written as $\mathcal{E}(B)$. Elements of $\mathcal{E}(B)$ will be written as $\left(B[t], m_{t}, \Delta_{t}\right)$.

Two $l$-deformations $\left(B[t] /\left(t^{l+1}\right), m_{t}^{l}, \Delta_{t}^{l}\right)$ and $\left(B[t] /\left(t^{l+1}\right),{m^{\prime}}_{t}^{l}, \Delta^{\prime l}\right)$ are said to be isomorphic, if there exists an isomorphism of $\mathbb{K}[t] /\left(t^{l+1}\right)$-bialgebras

$$
\phi:\left(B[t] /\left(t^{l+1}\right), m_{t}^{l}, \Delta_{t}^{l}\right) \longrightarrow\left(B[t] /\left(t^{l+1}\right), m_{t}^{\prime l}, \Delta_{t}^{\prime l}\right)
$$

such that $\phi$ is homogeneous of degree zero and

$$
\phi \equiv \operatorname{Id}_{B} \otimes \operatorname{Id}_{\mathbb{K}[t] /\left(t^{l+1}\right)} \bmod (t) .
$$

Denote by

$$
i s o \mathcal{E}^{l}(B) \quad(\text { resp. } \quad i s o \mathcal{E}(B))
$$

the set of isoclasses of $l$-deformations (resp. deformations) of the graded bialgebra $B$, for $l \in \mathbb{N}$. 
2.3. Use the notation as above. Consider an element $\left(B[t] /\left(t^{l+1}\right), m_{t}^{l}, \Delta_{t}^{l}\right)$ of $\mathcal{E}^{l}(B)$. By definition, we can write

$$
m_{t}^{l}(a \otimes b)=\sum_{0 \leq s \leq l} m_{s}(a \otimes b) t^{s}
$$

and

$$
\Delta_{t}^{l}(c)=\sum_{0 \leq s \leq l} \Delta_{s}(c) t^{s}
$$

where $a, b, c \in B$, and $m_{s}: B \otimes B \longrightarrow B$ and $\Delta_{s}: B \longrightarrow B \otimes B$ are homogeneous of degree $-s$. Note that $m_{0}=m$ and $\Delta_{0}=\Delta$.

It is easy to check that the associativity of $m_{t}^{l}$, the compatibility of $m_{t}^{l}$ and $\Delta_{t}^{l}$, and the coassociativity of $\Delta_{t}^{l}$ are equivalent to the following identities, respectively, for each $1 \leq n \leq l$,

$$
\begin{gathered}
a m_{n}(b \otimes c)-m_{n}(a b \otimes c)+m_{n}(a \otimes b c)-m_{n}(a \otimes b) c \\
=\sum_{1 \leq s \leq n-1} m_{s}\left(m_{n-s}(a \otimes b) \otimes c\right)-m_{s}\left(a \otimes m_{n-s}(b \otimes c)\right), \\
m_{n}\left(a_{(1)} \otimes b_{(1)}\right) \otimes a_{(2)} b_{(2)}-\Delta\left(m_{n}(a \otimes b)\right)+a_{(1)} b_{(1)} \otimes m_{n}\left(a_{(2)} \otimes b_{(2)}\right) \\
+a_{(1)} b_{l} \otimes a_{(2)} b_{r}-\Delta_{n}(a b)+a_{l} b_{(1)} \otimes a_{r} b_{(2)} \\
=-\sum_{1 \leq s, r, s^{\prime}, r^{\prime} \leq n, s+s^{\prime}+r+r^{\prime}=n}\left(m_{r} \otimes m_{r^{\prime}}\right) \circ \tau_{23} \circ\left(\Delta_{s} \otimes \Delta_{s^{\prime}}\right)(a \otimes b),
\end{gathered}
$$

and

$$
\begin{aligned}
& c_{(1)} \otimes \Delta_{n}\left(c_{(2)}\right)-(\Delta \otimes \mathrm{Id}) \circ \Delta_{n}(c)+(\mathrm{Id} \otimes \Delta) \circ \Delta_{n}(c)-\Delta_{n}\left(c_{(1)}\right) \otimes c_{(2)} \\
= & \sum_{1 \leq s \leq n-1}\left(\Delta_{n-s} \otimes \mathrm{Id}\right) \circ \Delta_{s}(c)-\left(\operatorname{Id} \otimes \Delta_{n-s}\right) \circ \Delta_{s}(c),
\end{aligned}
$$

where we use Sweedler's notation $\Delta(a)=a_{(1)} \otimes a_{(2)}, a \in B$, and in the second identity we use the notation $\Delta_{n}(a)=a_{l} \otimes a_{r}$ and $\Delta_{n}(b)=b_{l} \otimes b_{r}$, and the map $\tau_{23}$ is the canonical flip map at the second and third positions.

Let $\left(B[t] /\left(t^{l+1}\right), m_{t}^{l}, \Delta_{t}^{l}\right)$ and $\left(B[t] /\left(t^{l+1}\right), m_{t}^{l}, \Delta_{t}^{l}\right)$ be two $l$-deformations with the maps $m_{s}, \Delta_{s}$ and $m_{s}^{\prime}, \Delta_{s}^{\prime}$ as in (2.1) and (2.2). An isomorphism $\phi$ between these deformations is given by

$$
\phi(a)=\sum_{0 \leq s \leq l} \phi_{s}(a) t^{s}, \quad a \in B
$$

where $\phi_{s}: B \longrightarrow B$ is a homogeneous map of degree $-s$. Note that $\phi_{0}=$ $\operatorname{Id}_{B}$. The facts that $\phi$ is a morphism of $\mathbb{K}[t] /\left(t^{l+1}\right)$-bialgebras implies $\phi$ 
preserves the identity element $1_{B}$ and the counit $\varepsilon_{t}^{l}$, and it satisfies, for each $1 \leq n \leq l$,

$$
\begin{aligned}
& \left(m_{n}-m_{n}^{\prime}\right)(a \otimes b)=a \phi_{n}(b)-\phi_{n}(a b)+\phi_{n}(a) b \\
& \quad+\sum_{0<s<n}\left\{\phi_{s}(a) \phi_{n-s}(b)-\phi_{s}\left(m_{n-s}(a \otimes b)\right)+\sum_{r+r^{\prime}=n-s} m_{s}^{\prime}\left(\phi_{r}(a) \otimes \phi_{r^{\prime}}(b)\right)\right\}
\end{aligned}
$$

and

$$
\begin{aligned}
& \left(\Delta_{n}-\Delta_{n}^{\prime}\right)(c)=\Delta\left(\phi_{n}(c)\right)-c_{(1)} \otimes \phi_{n}\left(c_{(2)}\right)-\phi_{n}\left(c_{(1)}\right) \otimes c_{(2)} \\
& \quad+\Delta_{s}\left(\phi_{n-s}(c)\right)-\sum_{0<s<n}\left\{\left(\phi_{s} \otimes \phi_{n-s}\right)(\Delta(c))-\sum_{r+r^{\prime}=n-s}\left(\phi_{r} \otimes \phi_{r^{\prime}}\right)\left(\Delta_{s}^{\prime}(c)\right)\right\},
\end{aligned}
$$

for all $a, b, c \in B$. Note that above discussion works for all $l \in \mathbb{N} \cup\{+\infty\}$.

The analogue of the following lemma is well-known in classical deformation theory.

Lemma 2.1. There exist restriction maps $r_{l, l^{\prime}}: \mathcal{E}^{l}(B) \longrightarrow \mathcal{E}^{l^{\prime}}(B)$ for every $l>l^{\prime} \in \mathbb{N}$, and maps $r_{l}: \mathcal{E}(B) \longrightarrow \mathcal{E}^{l}(B)$ such that

$$
\mathcal{E}(B)=\lim _{l \in \mathbb{N}} \mathcal{E}^{l}(B) \text {. }
$$

Proof. The restriction map $r_{l, l^{\prime}}$ is given as follows: given $\left(B[t] /\left(t^{l+1}\right), m_{t}^{l}, \Delta_{t}^{l}\right)$ in $\mathcal{E}^{l}(B)$ with the maps $m_{s}$ and $\Delta_{s}$ defined in (2.1) and (2.2), just define $m_{t}^{l^{\prime}}:=\sum_{0 \leq s \leq l^{\prime}} m_{s} t^{s}$ and $\Delta_{t}^{l^{\prime}}:=\sum_{0 \leq s \leq l^{\prime}} \Delta_{s} t^{s}$; it is direct to check that $\left(B[t] /\left(t^{l^{\prime}+1}\right), m_{t}^{l^{\prime}}, \Delta_{t}^{l^{\prime}}\right)$ is the desired element in $\mathcal{E}^{l^{\prime}}(B)$. The map $r_{l}$ is defined in a similar way, and then the result is obvious.

A graded bialgebra $B=\oplus_{n \geq 0} B_{(n)}$ is called graded-rigid if the set $i s o \mathcal{E}(B)$ has only one element, i.e., any deformation of $B$ is isomorphic to the trivial one.

2.4. We have the following observation, which says that the graded bialgebra deformations coincide with the liftings.

Theorem 2.2. Let $B=\oplus_{n \geq 0} B_{(n)}$ be a graded bialgebra. There exists a natural bijection

$$
\operatorname{Lift}(B) \simeq \operatorname{iso\mathcal {E}}(B) .
$$

Proof. We will construct a map $F: \operatorname{Lift}(B) \longrightarrow i \operatorname{so\mathcal {E}}(B)$. Given a lifting $U$ of $B$. Denote by $m_{U}$ and $\Delta_{U}$ the multiplication and comultiplication maps of $U$. Since $U$ is a filtered bialgebra, we have

$$
m_{U}: B_{i} \otimes B_{j} \longrightarrow B_{i+j} \quad \text { and } \quad \Delta_{U}: B_{n} \longrightarrow \sum_{i+j=n} B_{i} \otimes B_{j} .
$$


Therefore, there uniquely exist homogeneous maps of degree $-s, m_{s}: B \otimes$ $B \longrightarrow B$ and $\Delta_{s}: B \longrightarrow B \otimes B$, such that

$$
m_{U}(a \otimes b)=\sum_{s \geq 0} m_{s}(a \otimes b) \quad \text { and } \quad \Delta_{U}(c)=\sum_{s \geq 0} \Delta_{s}(c) .
$$

By $\operatorname{gr} U=B$ as graded bialgebras, we have $m_{0}=m$ and $\Delta_{0}=\Delta$.

Now Define $F(U)=\left(B[t], m_{t}, \Delta_{t}\right)$ as follows

$$
m_{t}(a \otimes b):=\sum_{s \geq 0} m_{s}(a \otimes b) t^{s} \quad \text { and } \quad \Delta_{t}(c):=\sum_{s \geq 0} \Delta_{s}(c) t^{s} .
$$

It is direct to check that $F(U)$ is a deformation.

The map $F$ is well-defined, i.e., it sends equivalent liftings to isomorphic deformations. In fact, given two liftings $U$ and $V$, an equivalence $\theta$ is a filtered isomorphism, hence there uniquely exist homogeneous maps $\phi_{s}$ : $B \longrightarrow B$ of degree $-s$ such that

$$
\theta(a)=\sum_{s \geq 0} \phi_{s}(a), \quad a \in B
$$

Then define $\phi: B[t] \longrightarrow B[t]$ to be $\mathbb{K}[t]$-linear such that by $\phi(a)=\sum_{s>0} \phi_{s}(a) t^{s}$. Hence $\phi$ is an isomorphism between the deformations $F(U)$ and $F(\bar{V})$.

On the other hand, by seeing (2.1) and (2.2), one obtains that $F$ is a bijection. This completes the proof.

An immediate consequence of Theorem 2.2 is

Corollary 2.3. Let $B=\oplus_{n \geq 0} B_{(n)}$ be a graded bialgebra. Then $B$ is gradedrigid implies that, for any filtered bialgebra $U$ such that $\operatorname{gr} U \simeq B$ as graded bialgebras, we have $U \simeq B$ as bialgebras.

If we assume that $B$ is coradically-graded, the converse is also true.

Proof. By Theorem 2.2, B is graded-rigid if and only if $\operatorname{Lift}(B)$ is a single element set, i.e., every lifting of $B$ is trivial.

Fir the first statement, such filtered bialgebra $U$ with $\operatorname{gr} U \simeq B$ gives rise to a lifting on $B$, denoted by $U^{\prime}$, such that $U \simeq U^{\prime}$ (as bialgebras). Since $B$ is graded-rigid, we get $U^{\prime} \simeq B$, thus we are done.

For the second one, assume $B$ is coradically-graded. Let $U$ be a lifting of $B$. Thus by the assumption, there exists an isomorphism $\theta: U \simeq B$. Note that $\theta$ preserves the coradical filtration, thus $\operatorname{gr} \theta$ can be viewed as a graded automorphism of $B$. Thus take $\theta^{\prime}=(\operatorname{gr} \theta)^{-1} \circ \theta: U \simeq B$. So $\theta^{\prime}$ realizes an equivalence between the lifting $U$ and the trivial lifting. This proves that $B$ is graded-rigid.

2.5. In this subsection, we assume that the base field $\mathbb{K}$ is algebraically closed of characteristic zero. One can define the variety Bialg ${ }_{n}$ of the bialgebra structures on $n$-dimensional spaces, which carries a natural $G L_{n}(\mathbb{K})$ action by base changes, see [10] and [7]. Recall that a bialgebra $B$ is called 
rigid if $G L_{n}(\mathbb{K})$-oribit of Bialg ${ }_{n}$ containing $B$ is Zariski open. In fact, we have

Corollary 2.4. Let $\mathbb{K}$ be an algebraically closed field of characteristic zero, $B=\oplus_{n \geq 0} B_{(n)}$ a finite dimensional graded bialgebra over $\mathbb{K}$. If $B$ is rigid, then $B$ is graded-rigid in the sense of $\mathbf{2 . 3}$.

Proof. By Corollary 2.3, we only need to show that every filtered bialgebra $U$ with $\operatorname{gr} U \simeq B$ is isomorphic to $B$. Assume the dimension of $B$ is $n$. By Theorem 3.4 in [7, $B$ is a degeneration of $U$, i.e., lies the closure of the orbit of $U$ (in the variety $\operatorname{Bialg}_{n}$ ). However the $G L_{n}(\mathbb{K})$-orbit of $B$ is open, we obtain that $B$ and $U$ belong to the same $G L_{n}(\mathbb{K})$-orbit, i.e., $B \simeq U$ as bialgebras, finishing the proof.

\section{Graded Bialgebra Cohomology}

In this section we will relate the graded bialgebra deformations with corresponding cohomology groups, which will be a graded (and normalized) version of "hat" bialgebra cohomology groups introduced in [5] (also see 9]).

3.1. Let $(B, m, e, \Delta, \varepsilon)$ be a bialgebra. Again we will use Sweedler's notation $\Delta(a)=a_{(1)} \otimes a_{(2)}, a \in B$.

Let us recall the bicomplex in [5] or 9], p.619. For this end, we need the following maps, where $p, q \geq 1$ and all $b$ 's are in $B, \lambda^{p}: B^{\otimes p+1} \longrightarrow B^{\otimes p}$ and $\rho^{p}: B^{\otimes p+1} \longrightarrow B^{\otimes p}$ given by

$$
\begin{aligned}
& \lambda^{p}\left(b^{1} \otimes \cdots \otimes b^{p+1}\right)=b_{(1)}^{1} b^{2} \otimes \cdots \otimes b_{(p)}^{1} b^{p+1}, \\
& \rho^{p}\left(b^{1} \otimes \cdots \otimes b^{p+1}\right)=b^{1} b_{(1)}^{p+1} \otimes \cdots \otimes b^{p} b_{(p)}^{p+1} .
\end{aligned}
$$

Dually, the maps $\sigma^{q}: B^{\otimes q} \longrightarrow B^{\otimes q+1}$ and $\tau^{q}: B^{\otimes q} \longrightarrow B^{\otimes q+1}$ are given by

$$
\begin{aligned}
& \sigma^{q}\left(b^{1} \otimes \cdots \otimes b^{q}\right)=\left(b_{(1)}^{1} \cdots b_{(1)}^{q}\right) \otimes b_{(2)}^{1} \otimes \cdots b_{(2)}^{q}, \\
& \tau^{q}\left(b^{1} \otimes \cdots \otimes b^{q}\right)=b_{(1)}^{1} \otimes \cdots \otimes b_{(1)}^{q} \otimes\left(b_{(2)}^{1} \cdots b_{(2)}^{q}\right) .
\end{aligned}
$$

In addition, we need $\Delta_{i}^{p}: B^{\otimes p} \longrightarrow B^{\otimes p+1}$ and $\mu_{j}^{q}: B^{\otimes q+1} \longrightarrow B^{\otimes q}, 1 \leq i \leq$ $p$ and $1 \leq j \leq q$, which are given by

$$
\begin{aligned}
& \Delta_{i}^{p}\left(b^{1} \otimes \cdots \otimes b^{p}\right)=b^{1} \otimes \cdots \otimes b_{(1)}^{i} \otimes b_{(2)}^{i} \otimes \cdots \otimes b^{p}, \\
& \mu_{i}^{q}\left(b^{1} \otimes \cdots \otimes b^{q+1}\right)=b^{1} \otimes \cdots \otimes b^{i} b^{i+1} \otimes \cdots \otimes b^{q+1} .
\end{aligned}
$$

Let $C^{p, q}=\operatorname{Hom}_{\mathbb{K}}\left(B^{\otimes q}, B^{\otimes p}\right), p, q \geq 1$. Define

$$
\delta_{h}^{p, q}: C^{p, q} \longrightarrow C^{p, q+1} \quad \text { and } \quad \delta_{c}^{p, q}: C^{p, q} \longrightarrow C^{p+1, q}
$$


which are given by

$$
\begin{aligned}
& \delta_{h}^{p, q}(f)=\lambda^{p} \circ(\mathrm{Id} \otimes f)+\sum_{i=1}^{q}(-1)^{i} f \circ \mu_{i}^{q}+(-1)^{q+1} \rho^{p} \circ(f \otimes \mathrm{Id}) \\
& \delta_{c}^{p, q}(f)=(\operatorname{Id} \otimes f) \circ \sigma^{q}+\sum_{j=1}^{p}(-1)^{j} \Delta_{j}^{p} \circ f+(-1)^{p+1}(f \otimes \mathrm{Id}) \circ \tau^{q}
\end{aligned}
$$

for $f \in C^{p, q}$, where Id denotes the identity map of $B$.

It is direct to check that $\left(C^{p, q}, \delta_{h}^{p, q}, \delta_{c}^{p, q}\right)$ is a bicomplex (see 9], p.619), i.e.,

$$
\delta_{h}^{p, q+1} \circ \delta_{h}^{p, q}=0, \quad \delta_{c}^{p, q+1} \circ \delta_{h}^{p, q}=\delta_{h}^{p+1, q} \circ \delta_{c}^{p, q}, \quad \delta_{c}^{p+1, q} \circ \delta_{c}^{p, q}=0 .
$$

We will introduce a sub bicomplex of the above bicomplex. Let $\mathrm{m}=$ Ker $\varepsilon$. Denote by $\mathrm{i}: \mathrm{m} \longrightarrow B$ the inclusion map, and $\pi: B \longrightarrow \mathrm{m}$ is given by $\pi(b)=b-\varepsilon(b) 1_{B}, b \in B$. Set $D^{p, q}=\operatorname{Hom}_{\mathbb{K}}\left(\mathrm{m}^{\otimes q}, \mathrm{~m}^{\otimes p}\right), p, q \geq 1$. Note that we have a natural embedding $D^{p, q} \hookrightarrow C^{p, q}$ by identifying

$$
f \in D^{p, q} \quad \text { with } \quad i^{\otimes p} \circ f \circ \pi^{\otimes q} \in C^{p, q} .
$$

We have the following observation

Lemma 3.1. Use the above notation. Then $\delta_{h}^{p, q}\left(D^{p, q}\right) \subseteq D^{p, q+1}$ and $\delta_{c}^{p, q}\left(D^{p, q}\right) \subseteq$ $D^{p+1, q}$.

Proof. Just note that $g \in C^{p, q}$ lies in $D^{p, q}$ if and only if

$$
\left(\mathrm{Id}^{\otimes j-1} \otimes \varepsilon \otimes \mathrm{Id}^{\otimes p-j}\right) \circ f=0
$$

and

$$
f\left(b^{1} \otimes \cdots \otimes b^{i-1} \otimes 1 \otimes b^{i+1} \otimes \cdots \otimes b^{q}\right)=0,
$$

for any $1 \leq i \leq q, 1 \leq j \leq p$ and any $b^{i} \in B$. Then the lemma follows from the definition of $\delta_{h}^{p, q}$ and $\delta_{c}^{p, q}$ immediately.

3.2. From now on $B=\oplus_{n \geq 0} B_{(n)}$ will be a graded bialgebra. In this case $\mathrm{m} \subseteq B$ is a graded subspace. Consider $D_{(l)}^{p, q}:=\operatorname{Hom}_{\mathbb{K}}\left(\mathrm{m}^{\otimes q}, \mathrm{~m}^{\otimes p}\right)_{(l)}, l \in \mathbb{Z}$, whose elements are homogeneous maps from $\mathrm{m}^{\otimes q}$ to $\mathrm{m}^{\otimes p}$ of degree $l$. Note that $D_{(l)}^{p, q} \subseteq D^{p, q} \hookrightarrow C^{p, q}$. We have the following

Lemma 3.2. $\delta_{h}^{p, q}\left(D_{(l)}^{p, q}\right) \subseteq D_{(l)}^{p, q+1}$ and $\delta_{c}^{p, q}\left(D_{(l)}^{p, q}\right) \subseteq D_{(l)}^{p+1, q}$ for each $l \in \mathbb{Z}$, $p, q \geq 1$.

Proof. Set $C_{(l)}^{p, q}=\operatorname{Hom}_{\mathbb{K}}\left(B^{\otimes q}, B^{\otimes p}\right)_{(l)}$. Clearly, $D_{(l)}^{p, q}=D^{p, q} \cap C_{(l)}^{p, q}$. From the definition of $\delta_{h}^{p, q}$ and $\delta_{c}^{p, q}$, one sees that they preserve the degrees, i.e. ,$\delta_{h}^{p, q}\left(C_{(l)}^{p, q}\right) \subseteq C_{(l)}^{p, q+1}$ and $\delta_{c}^{p, q}\left(C_{(l)}^{p, q}\right) \subseteq C_{(l)}^{p+1, q}$. Now the result follows from Lemma 3.1. 
Denote by $\delta_{h,(l)}^{p, q}\left(\operatorname{resp} . \delta_{c,(l)}^{p, q}\right)$ the restriction of the maps $\delta_{h}^{p, q}\left(\right.$ resp. $\left.\delta_{c}^{p, q}\right)$ to the subspace $D_{(l)}^{p, q}$. Thus by Lemma 3.2 , we get a bicomplex $\left(D_{(l)}^{p, q}, \delta_{h,(l)}^{p, q}, \delta_{c,(l)}^{p, q}\right)$ for each $l \in \mathbb{Z}$.

There is a canonical way to construct a complex from a given bicomplex: set

$$
\hat{D}_{(l)}^{n}=\bigoplus_{p+q=n+1, p, q \geq 1} D_{(l)}^{p, q}, \quad n \geq 1
$$

define $\partial_{(l)}^{n}: \hat{D}_{(l)}^{n} \longrightarrow \hat{D}_{(l)}^{n+1}$ by

$$
\left.\partial_{(l)}^{n}\right|_{D_{(l)}^{n+1-q, q}}:=\delta_{h,(l)}^{p, q}+(-1)^{q} \delta_{c,(l)}^{p, q}, \quad 1 \leq q \leq n .
$$

Hence, for each $l \in \mathbb{Z}$, we get a complex

$$
0 \longrightarrow \hat{D}_{(l)}^{1} \stackrel{\partial_{(l)}^{1}}{\longrightarrow} \hat{D}_{(l)}^{2} \stackrel{\partial_{(l)}^{2}}{\longrightarrow} \hat{D}_{(l)}^{3} \stackrel{\partial_{(l)}^{3}}{\longrightarrow} \hat{D}_{(l)}^{4} \longrightarrow \cdots
$$

We define the n-th cohomology group of the above complex to be the $n$-th graded "hat" bialgebra cohomology of degree $l$ of the graded bialgebra $B$, which will be denoted by $\hat{h}_{b}^{n}(B)_{(l)}, n \geq 1, l \in \mathbb{Z}$.

It is very useful to write out $\hat{h}_{b}^{2}(B)_{(l)}$ and $\hat{h}_{b}^{3}(B)_{(l)}$ explicitly from the definition. (In what follows, we will use the maps $\delta_{h}^{p, q}$ and $\delta_{c}^{p, q}$, instead of $\delta_{h,(l)}^{p, q}$ and $\left.\delta_{c,(l)}^{p, q}\right)$

(1). The cohomology group $\hat{h}_{b}^{2}(B)_{(l)}$ consists of all pairs $(f, g)$, where $f$ : $\mathrm{m} \otimes \mathrm{m} \longrightarrow \mathrm{m}$ and $g: \mathrm{m} \longrightarrow \mathrm{m} \otimes \mathrm{m}$ are homogeneous maps of degree $l$, satisfying the following relations:

$$
\delta_{h}^{1,2}(f)=0, \quad \delta_{c}^{1,2}(f)-\delta_{h}^{2,1}(g)=0, \quad \delta_{c}^{2,1}(g)=0,
$$

i.e., for any $a, b, c \in \mathrm{m}$, we have

$$
\begin{aligned}
& a f(b \otimes c)-f(a b \otimes c)+f(a \otimes b c)-f(a \otimes c) b=0, \\
& f\left(a_{(1)} \otimes b_{(1)}\right) \otimes a_{(2)} b_{(2)}-\Delta(f(a \otimes b))+a_{(1)} b_{(1)} \otimes f\left(a_{(2)} \otimes b_{(2)}\right) \\
& +a_{(1)} g(b)_{l} \otimes a_{(2)} g(b)_{r}-g(a b)+g(a)_{l} b_{(1)} \otimes g(a)_{r} b_{(2)}=0, \\
& c_{(1)} \otimes g\left(c_{(2)}\right)-(\Delta \otimes \operatorname{Id})(g(c))+(\operatorname{Id} \otimes \Delta)(g(c))-g\left(c_{(1)}\right) \otimes c_{(2)}=0 \text {, }
\end{aligned}
$$

where we write $g(b)=g(b)_{l} \otimes g(b)_{r}, b \in B$.

Two pairs $(f, g)=\left(f^{\prime}, g^{\prime}\right)$ in $\hat{h}_{b}^{2}(B)_{(l)}$ if and only if there exists a homogeneous map $\theta: \mathrm{m} \longrightarrow \mathrm{m}$ of degree $l$ such that, for any $a, b, c \in \mathrm{m}$,

$$
\begin{aligned}
& \left(f-f^{\prime}\right)(a \otimes b)=a \theta(b)-\theta(a b)+\theta(a) b, \\
& \left(g-g^{\prime}\right)(c)=\Delta(\theta(c))-c_{(1)} \otimes \theta\left(c_{(2)}\right)-\theta\left(c_{(1)}\right) \otimes c_{(2)} .
\end{aligned}
$$


(2). The group $\hat{h}_{b}^{3}(B)_{(l)}$ consists of all triples $(F, H, G)$, where $F: \mathrm{m} \otimes \mathrm{m} \otimes \mathrm{m} \longrightarrow \mathrm{m}, \quad H: \mathrm{m} \otimes \mathrm{m} \longrightarrow \mathrm{m} \otimes \mathrm{m}, \quad G: \mathrm{m} \longrightarrow \mathrm{m} \otimes \mathrm{m} \otimes \mathrm{m}$ are homogeneous maps of degree $l$, subject to the relations:

$$
\delta_{h}^{1,3}(F)=0, \quad \delta_{h}^{2,2}(F)=\delta_{c}^{1,3}(H), \quad \delta_{c}^{2,2}(H)=-\delta_{h}^{1,3}(G), \quad \delta_{c}^{3,1}(G)=0 .
$$

Note that $(F, H, G)=0$ in $\hat{h}_{b}^{3}(B)_{(l)}$ if and only if there exists $(f, g) \in \hat{D}_{(l)}^{2}$ such that

$$
(F, G, H)=\partial_{(l)}^{2}((f, g)),
$$

which can be written out in the component form just by the definition of $\partial_{(l)}^{2}$

3.3. Now we are at the position to present our main observations, which relates the graded bialgebra deformations of the graded bialgebra $B$ with the cohomology groups $\hat{h}_{b}^{2}(B)_{(l)}$ and $\hat{h}_{b}^{3}(B)_{(l)}$.

Theorem 3.3. Let $B=\oplus_{n \geq 0} B_{(n)}$ be a graded bialgebra. Use the notation as above. Then

(1) There is a bijection between iso $\mathcal{E}^{1}(B)$ and $\hat{h}_{b}^{2}(B)_{(-1)}$.

(2) If $\hat{h}_{b}^{2}(B)_{(-l)}=0$ for each $l \geq 1$, then the graded bialgebra $B$ is gradedrigid.

(3) The obstruction to extend an element of $\mathcal{E}^{l}(B)$ to $\mathcal{E}^{l+1}(B)$ lies in $\hat{h}_{b}^{3}(B)_{(-l-1)}, l \geq 1$.

Proof. (1) Recall from $\mathbf{2 . 2}$ that an element in $\mathcal{E}^{1}(B)$ is just given by $\left(B[t] /\left(t^{2}\right), m_{t}^{2}, \Delta_{t}^{2}\right)$. As in 2.3, write

$$
m_{t}^{2}(a \otimes b)=a b+f(a \otimes b) t, \quad \Delta_{t}^{2}(c)=\Delta(c)+g(c) t,
$$

where $f: B \otimes B \longrightarrow B$ and $g: B \longrightarrow B \otimes B$ are homogeneous of degree -1 . Note that $1_{B}$ is the identity element of $B[t] /\left(t^{2}\right)$, hence $f\left(1_{B} \otimes b\right)=$ $f\left(b \otimes 1_{B}\right)=0$ for all $b \in B$. Moreover, for $a, b \in \mathrm{m}, \varepsilon_{t}\left(m_{t}^{2}(a \otimes b)\right)=0$ implies that $\varepsilon_{t}(a b+f(a \otimes b) t)=0$, i.e., $f(a \otimes b) \in \mathrm{m}$. Thus we may view $f$ belongs to $D_{(-1)}^{1,2}$. Dually one can show that $g \in D_{(-1)}^{2,1}$.

Note that $m_{t}^{2}$ is an associative multiplication on $B[t] /\left(t^{2}\right)$, thus we get

$$
f(a \otimes b) c-f(a b \otimes) c+f(a \otimes b c)-a f(b \otimes c)=0, \quad \forall a, b, c \in B .
$$

Therefore we get equation (3.1). Similarly, the fact that $\Delta_{t}^{2}$ is an algebra morphism (resp. that $\Delta_{t}^{2}$ is an coassociative comultiplication) gives us equation $(3.2)$ (resp. equation (3.3)), i.e., $(f, g)$ can be viewed as an element in $\hat{h}_{b}^{2}(B)_{(-1)}$.

Suppose that $\left(B[t] /\left(t^{2}\right), m_{t}^{2}, \Delta_{t}^{2}\right)$ and $\left(B[t] /\left(t^{2}\right),{m^{\prime}}_{t}{ }^{2} \Delta^{\prime 2}\right)$ are two isomorphic deformations, with $(f, g)$ and $\left(f^{\prime}, g^{\prime}\right)$ defined as above, respectively. Let 
$\phi$ (see also 2.3) be the isomorhism. We may write

$$
\phi(a)=a+\theta(a) t, \quad \forall a \in B
$$

for some homogeneous map $\theta: B \longrightarrow B$ of degree -1 . (Note that the map $\theta$ may be viewed as a map from $\mathrm{m}$ to $\mathrm{m}$.) Now it is direct to check that $\theta$ realizes an equivalence of $(f, g)$ and $\left(f^{\prime}, g^{\prime}\right)$ in $\hat{h}_{b}^{3}(B)_{(l)}$. Now we have establish a map from $\mathcal{E}^{1}(B)$ to $\hat{h}_{b}^{2}(B)_{(-1)}$, sending $\left(B[t] /\left(t^{2}\right), m_{t}^{2}, \Delta_{t}^{2}\right)$ to $(f, g)$. One can easily see that the correspondence is bijective, as required.

(2) To prove that $B$ is graded-rigid, we just need to show that $i s o \mathcal{E}(B)$ is a single-element set.

Let $\left(B[t], m_{t}, \Delta_{t}\right)$ be an element in $\mathcal{E}(B)$. As before, write

$$
m_{t}(a \otimes b)=\sum_{s=0}^{\infty} m_{s}(a \otimes b) t^{s} \quad \text { and } \quad \Delta_{t}(c)=\sum_{s=0}^{\infty} \Delta_{s}(c) t^{s} .
$$

Note that $m_{0}=m$ and $\Delta_{0}=\Delta$, and $m_{s}$ and $\Delta_{s}$ are homogeneous maps of degree $-s$. By a similar argument as (1), we may view $m_{s} \in D_{(-s)}^{1,2}$ and $\Delta_{s} \in D_{(-s)}^{2,1}$. Moreover, from (1), we see that $\left(m_{1}, \Delta_{1}\right)$ can be viewed as an element in $\hat{h}_{b}^{1}(B)_{(-1)}$. Now by the assumption, there exists a homogeneous map $\theta_{1}: \mathrm{m} \longrightarrow \mathrm{m}$ of degree -1 , such that (see (3.4) and (3.5))

$$
\begin{aligned}
& m_{1}(a \otimes b)=a \theta_{1}(b)-\theta_{1}(a b)+\theta_{1}(a) b, \\
& \Delta_{1}(c)=\Delta\left(\theta_{1}(c)\right)-c_{(1)} \otimes \theta_{1}\left(c_{(2)}\right)-\theta_{1}\left(c_{(1)}\right) \otimes c_{(2)} .
\end{aligned}
$$

Take $\phi_{1}: B[t] \longrightarrow B[t]$ to be a $\mathbb{K}[t]$-linear map such that

$$
\phi_{1}(a)=a+\theta(a) t, \quad a \in B .
$$

Note that $\phi_{1}$ is a bijective map preserving the identity $1_{B}$ and the counit $\varepsilon_{t}$. Consider a deformation

$$
\left(B[t], m_{t}^{\prime}=\phi_{1} \circ m_{t} \circ\left(\phi_{1}^{-1} \otimes \phi_{1}^{-1}\right), \Delta_{t}^{\prime}=\left(\phi_{1} \otimes \phi_{1}\right) \circ \Delta_{t} \circ \phi_{1}^{-1}\right) .
$$

We have

$$
\begin{aligned}
m_{t}^{\prime}(a \otimes b) & =a b+m_{2}^{\prime}(a \otimes b) t^{2}+m_{3}^{\prime}(a \otimes b) t^{3}+\cdots, \\
\Delta_{t}^{\prime}(c) & =\Delta(c)+\Delta_{2}^{\prime}(c) t^{2}+\Delta_{2}^{\prime}(c), t^{3}+\cdots
\end{aligned}
$$

where $m_{s}{ }^{\prime}$ and $\Delta_{s}{ }^{\prime}$ are homogeneous maps of degree $-s, s \geq 2$. Now by comparing (2.3-5) and (3.1-3), we see that $\left(m_{2}{ }^{\prime}, \Delta_{2}{ }^{\prime}\right)$ can be viewed as an element in $\hat{h}_{b}^{2}(B)_{(-2)}$. Hence there exists a homogeneous map $\theta_{2}: \mathrm{m} \longrightarrow \mathrm{m}$ of degree -2 , such that (again see (3.4) and (3.5))

$$
\begin{aligned}
& m_{2}^{\prime}(a \otimes b)=a \theta_{2}(b)-\theta_{2}(a b)+\theta_{2}(a) b \\
& \Delta_{2}^{\prime}(c)=\Delta\left(\theta_{2}(c)\right)-c_{(1)} \otimes \theta_{2}\left(c_{(2)}\right)-\theta_{2}\left(c_{(1)}\right) \otimes c_{(2)} .
\end{aligned}
$$

Take $\phi_{2}: B[t] \longrightarrow B[t]$ to be a $\mathbb{K}[t]$-linear map such that

$$
\phi_{2}(a)=a+\theta_{2}(a) t^{2}, \quad \forall a \in B .
$$


Now consider the following deformation

$$
\left(B[t], m_{t}^{\prime \prime}=\phi_{2} \circ m_{t}^{\prime} \circ\left(\phi_{2}^{-1} \otimes \phi_{2}^{-1}\right), \Delta_{t}^{\prime \prime}=\left(\phi_{2} \otimes \phi_{2}\right) \circ \Delta_{t}^{\prime} \circ \phi_{2}^{-1}\right),
$$

whose coefficients of $t$ and $t^{2}$ vanishes. Continue the above procedure, we can define $\theta_{3}$ and then $\phi_{3}, \cdots$.

Finally, define the infinite composition $\cdots \phi_{3} \circ \phi_{2} \circ \phi_{1}$ to be $\phi$. Note that the $\mathbb{K}[t]$-linear isomorphism $\phi: B[t] \longrightarrow B[t]$ is well-defined on every $a \in B$, which preserves the identity $1_{B}$ and the counit $\varepsilon_{t}$. By the construction of each map $\phi_{s}$, we obtain that the deformation $\left(B[t], \phi \circ m_{t} \circ\left(\phi^{-1} \otimes \phi^{-1}\right),(\phi \otimes\right.$ $\left.\phi) \circ \Delta_{t} \circ \phi^{-1}\right)$ is trivial, which is also equivalent to the given deformation. Thus we prove (2).

$$
\begin{aligned}
& \text { Let }\left(B[t] /\left(t^{l+1}\right), m_{t}^{l}, \Delta_{t}^{l}\right) \text { be an element in } \mathcal{E}^{l}(B) \text {. Write } \\
& \qquad m_{t}^{l}(a \otimes b)=\sum_{0 \leq s \leq l} m_{s}(a \otimes b) t^{s} \quad \text { and } \quad \Delta_{t}^{l}(c)=\sum_{0 \leq s \leq l} \Delta_{s}(c) t^{s}
\end{aligned}
$$

where $m_{s}$ and $\Delta_{s}$ are homogeneous maps of degree $-s$. By the same argument as above, one can show that $m_{s}\left(\right.$ resp. $\left.\Delta_{s}\right)$ can be viewed as maps from $\mathrm{m} \otimes \mathrm{m}$ to $\mathrm{m}$ (resp. from $\mathrm{m}$ to $\mathrm{m} \otimes \mathrm{m})$.

To extend $\left(B[t] /\left(t^{l+1}\right), m_{t}^{l}, \Delta_{t}^{l}\right)$ to some element in $\mathcal{E}^{l+1}(B)$, we just need to find some homogeneous maps $f: \mathrm{m} \otimes \mathrm{m} \longrightarrow \mathrm{m}$ and $g: \mathrm{m} \longrightarrow \mathrm{m} \otimes \mathrm{m}$ of degree $-(l+1)$ such that $\left(B[t] /\left(t^{l+2}\right), m_{t}^{l}+t^{l+1} f, \Delta_{t}^{l}+t^{l+1} g\right)$ is an bialgebra over $\mathbb{K}[t] /\left(t^{l+2}\right)$. Now the associativity of $m_{t}^{l}+t^{l+1} f$, the compatibility of the multiplication and comultiplication, and the coassociativity of $\Delta_{t}^{l}+t^{l+1} g$ gives exactly the following identities, respectively,

$$
\begin{aligned}
& F(a \otimes b \otimes c):=\sum_{s=1}^{l} m_{s}\left(m_{l+1-s}(a \otimes b) \otimes c\right)-m_{s}\left(a \otimes m_{l+1-s}(b \otimes c)\right) \\
& =a f(b \otimes c)-f(a b \otimes c)+f(a \otimes b c)-f(a \otimes b) c \\
& =\delta_{h}^{1,2}(f)(a \otimes b \otimes c), \\
& H(a \otimes b):=\sum_{s+r+s^{\prime}+r^{\prime}=l+1}\left(m_{s^{\prime}} \otimes m_{r^{\prime}}\right) \circ \tau_{23}\left(\Delta_{s}(a) \otimes \Delta_{r}(b)\right)-\sum_{s=1}^{l} \Delta_{s}\left(m_{l+1-s}(a \otimes b)\right) \\
& =\left(\delta_{c}^{1,2}(f)+\delta_{h}^{2,1}(g)\right)(a \otimes b),
\end{aligned}
$$

and

$$
\begin{aligned}
G(c): & =\sum_{s=1}^{l}\left(\Delta_{s} \otimes \mathrm{Id}\right) \circ \Delta_{l+1-s}(c)-\left(\mathrm{Id} \otimes \Delta_{s}\right) \circ \Delta_{l+1-s}(c) \\
& =c_{(1)} \otimes g\left(c_{(2)}\right)-(\Delta \otimes \mathrm{Id})(g(c))+(\operatorname{Id} \otimes \Delta)(g(c))-g\left(c_{(1)}\right) \otimes c_{(2)} \\
& =\delta_{c}^{2,1}(g)(c)
\end{aligned}
$$


where $a, b, c \in \mathrm{m}$, and $\tau_{23}$ is the flip map with respect to the second and third positions.

Now it is direct to check that the element $(F, H, G) \in \hat{D}_{(-l-1)}$ lies in the kernel of the differential $\partial_{(-l-1)}^{3}$ from (2.3-5), i.e., it can be viewed as an element in the cohomology group $\hat{h}_{b}^{3}(B)_{(-l-1)}$. Now by seeing the above three identities, we obtain that if $\hat{h}_{b}^{3}(B)_{(-l-1)}=0$, then such maps $f, g$ always exist, i.e., we can extend $\left(B[t] /\left(t^{l+1}\right), m_{t}^{l}, \Delta_{t}^{l}\right)$ to $\mathcal{E}^{l+1}(B)$. This completes the proof.

\section{REFERENCES}

[1] Andruskiewitsch, N., and Schneider, H.J., Lifting of quantum linear spaces and pointed Hopf algebras of order $p^{3}$, J. Algebra 209 (1998), 658-691.

[2] Andruskiewitsch, N., and Schneider, H.J., Pointed Hopf algebras, In "New directions in Hopf algebras", MSRI series Cambridge Univ. Press, (2002) 1-68.

[3] Braverman, A., and Gaitsgory, D., Poincaré-Birkhoff-Witt theorem for quadratic algebras of Koszul type, J. Algebra 181 (1996), 315-328.

[4] Gerstenhaber, M., Deformations of rings and algebras, Ann. of Math. (2) 79 (1964), 59-103.

[5] Gerstenhaber, M., and Schack, S.D., Bialgebra cohomology, deformations, and quantum groups, Proc. Natl. Acad. Sci. USA, vol.87 (1990), 478-481.

[6] Gerstenhaber, M., and Schack, S.D., Algebras, bialgberas, quantum groups and algebraic deformations, Contemporary Math. 134 (1992), 51-92.

[7] Makhlouf, A., Degeneration, rigidity and irreducible components of Hopf algebras, Algebra Colloqium, to appear. Also availabe at: math.RA/0211187

[8] Năstăsescu, C., and Oystaeyen, F. van, Methods of graded rings, Lecture Notes in Math., vol.1836, Springer-Verlag, Berlin, Heidelberg, 2004.

[9] Parshall, B., and Wang, J.P., On bialgebra cohomology, Bulletin De La Soc. Math. De Belgium, t.XLII (1990), 607-641.

[10] Stefan, D., The set of types of $n$-dimensional semisimple and cosemisimple Hopf algebras is finite, J.Algebra 193 (1997), 343-361.

[11] Sweedler, M. E., Hopf algebras, Benjamin, New York, 1969. 